Orange Journal / Volumen 3 Número 5/ Enero - junio 2021

DOI: https://doi.org/10.46502/issn.2710-995X/2021.5.03

Pérez Molinet, A., Hernándes Montero, F.E., Arencibia Castellanos, G., Rodriguez, J.R. (2021). Estimación de la posición a través de IMU basada en la detección de periodos de estabilidad durante la marcha. Orange Journal, 3(5), 16-29. https://doi.org/10.46502/issn.2710-995X/2021.5.03

\title{
Estimación de la posición a través de IMU basada en la detección de periodos de estabilidad durante la marcha
}

\section{Position estimation through IMU based on detection of stable periods during gait}

Recibido: 5 de agosto de 2021

Aceptado: 2 de septiembre de 2021

Escrito por:

Ailén Pérez Molinet ${ }^{6}$

https://orcid.org/0000-0001-9118-3495

Fidel Ernesto Hernándes Montero 7

https://orcid.org/0000-0002-5003-2807

Gianna Arencibia Castellanos ${ }^{8}$

https://orcid.org/0000-0001-6538-6707

Juan Raul Rodriguez ${ }^{9}$

https://orcid.org/0000-0002-9328-4537

\section{Resumen}

En esta investigación se presenta la propuesta de un algoritmo de estimación de posición para una aplicación de estimación de la longitud de la zancada en un proceso de marcha. La estimación de la posición se realiza mediante la detección de los periodos de estabilidad que están presente en una caminata. Para ello se emplearon sensores inerciales, específicamente acelerómetros y giroscopios. La propuesta fue validada mediante un trabajo con señales simuladas en presencia de diferentes condiciones El trabajo con las señales simuladas permitió llegar a conclusiones concretas a tener en cuenta, posteriormente, durante el trabajo con las señales reales. El procesamiento de ambas señales fue realizado en el software Matlab, el cual es conocido por sus bondades computacionales. A través de los diferentes experimentos realizados, se obtuvieron resultados con cierto grado de efectividad, determinada a través del promedio de la diferencia de la posición esperada con respecto a la posición estimada. El objetivo de esta investigación fue cumplido de manera satisfactoria.

Palabras clave: unidad de medición inercial, longitud de la zancada, estimación de gravedad, marcha humana.

\begin{abstract}
This work is focused to estimate position for an application of stride length estimation in a gait process. The position estimation is made by detecting walk stability periods. To do that, inertial sensors, specifically, accelerometers and gyroscopes were used. The proposal was validated by working with simulation signals, in a process that allowed for using the same algorithm parameters in the work with true signals. The goal position, or stride length, was estimated with a satisfactory effectiveness. The effectiveness was computed as the average of the difference of the expected and the estimated position.
\end{abstract}

Key Words: inertial measurement unit, stride length, gravity estimation, human gait.

\footnotetext{
${ }^{6}$ Máster en Ciencias, Jefa de Departamento de telecomunicaciones y Telemática, Grupo de Investigación Monitoreo de la Marcha, La Habana, Cuba.

${ }^{7}$ Doctor en Ciencias, Vicedecano de investigaciones, Grupo de Investigación Monitoreo de la Marcha, Habana, Cuba.

${ }^{8}$ Investigadora del Centro de Neurociencias de Cuba, Grupo de Investigación Monitoreo de la Marcha, La Habana, Cuba.

${ }^{9}$ Máster en Ciencias, Profesor de la Universidad de Pinar del Río, Grupo de Investigación Monitoreo de la Marcha, La Habana, Cuba.
} 


\section{ORANGE JOURNAL}

\section{Introducción}

La evaluación temprana de los signos y síntomas relacionados con el deterioro funcional contribuye a la detección de ancianos frágiles. Se ha comprobado que la detección precoz del anciano frágil y el empleo oportuno de técnicas diagnósticas, terapéuticas y rehabilitadoras pueden impactar positivamente en la calidad de vida del adulto mayor. Diversas son las investigaciones realizadas sobre la importancia de los indicadores del desempeño físico para la clasificación del grado de funcionalidad en los ancianos (Rantanen, Guralnik, Ferrucci, \& Leveille, 1999) (Gerety, et al., 1993). Entre los parámetros más utilizados para la evaluación del desempeño físico están los relacionados con la marcha (Loredo Medina, 2011).

Dentro de los métodos utilizados para realizar el análisis se encuentran los sensores inerciales. Actualmente el uso de sensores de aceleración se ha popularizado en el campo de la telemedicina (Pang \& Sing, Octubre 2011) debido al avance en las técnicas de diseño y fabricación que han conseguido miniaturizar y abaratar el coste de estos sensores.

En Cuba existe un antecedente de estudio de los indicadores del desempeño físico para caracterizar y determinar la fragilidad en adultos mayores (Garcia \& Bueno, 2010) (Garcia, PIinera, Garcia., \& Bueno, 2020) (Garcia, Morejon, De Dios, \& Rodriguez, 2018), y para priorizar su atención especializada en el Sistema Nacional de Salud. La evaluación de tales indicadores se ha realizado en el Centro de Investigación sobre Longevidad, Envejecimiento y Salud. Durante la evaluación, se miden, entre otros aspectos, parámetros de marcha, para lo cual se registran datos de velocidad, amplitud del paso, longitud de la zancada y cadencia. Este procedimiento se realiza por inspección visual y este carácter subjetivo ha propiciado que se busquen técnicas objetivas para la estimación de estos parámetros, y que permitan identificar más objetivamente a los ancianos vulnerables y con mayor riesgo de presentar discapacidad.

El desarrollo de un sistema electrónico basado en una unidad de medición inercial (IMU), es uno de los antecedentes con los que se cuenta para propiciar que el análisis de la marcha que se realiza en Cuba tenga un carácter más objetivo. Dicho sistema es capaz de almacenar las señales sensadas, que posteriormente serán empleadas para estimar los parámetros mencionados de la marcha. Dicho sistema dentro de su módulo de adquisición estuvo compuesto por un Arduino Nano, la unidad de medición inercial MPU-6050 (acelerómetros y giroscopios), un módulo Bluetooth utilizado para la transmisión inalámbrica de datos, un adaptador micro SD para el almacenamiento y una batería de $9 \mathrm{~V}$. El costo de este sistema desarrollado fue aproximadamente de 22 USD (Menendez Alvarez, 2018). Sin embargo, el mismo no proporciona de forma directa los parámetros que son analizados durante la marcha, como la longitud de la zancada, por lo que el análisis objetivo de la marcha aún sigue sin conseguirse. La longitud de la zancada, uno de los parámetros de la marcha, se puede estimar a través de técnicas de estimación de posición utilizando solamente el IMU que el sistema electrónico trae incorporado. Existen diversas técnicas para ello (Hannik et al, 2017) (Sijobert, Geny, \& Coste, 2015) (Martin, 2011) (Peruzzi, Della, \& Cereatti, 2011) (Vara Rodriguez, 2014) (Lee \& Lee, 2002); sin embargo, ninguna ha mostrado efectividad adecuada. Por tal motivo, el objetivo de este trabajo se dirigió a implementar un algoritmo de estimación de la longitud de la zancada, o lo que es lo mismo, estimación de la posición, empleando el sistema electrónico desarrollado.

\section{Marco Teórico}

Las unidades de medición inerciales son dispositivos electrónicos que combinan acelerómetros, giroscopios, y magnetómetros (brújulas), cuyo objetivo es obtener mediciones de velocidad, rotación y fuerzas gravitacionales (Vistronica, n.d.). Los acelerómetros y los giroscopios basados en Sistemas MicroElectro-Mecanizados (MEMS), se han convertido en los dispositivos más utilizados en el estudio del movimiento humano, ya que son de pequeño tamaño y peso, lo que facilita la no invasividad (Chen \& Basset, 2005) (Corder et al, 2008) (Kavanagh Menz, 2008).

Un giroscopio es un dispositivo electrónico capaz de medir la velocidad angular con respecto a un sistema de coordenadas rectangulares. Es usado en brújulas, sistemas de navegación o en dispositivos de reconocimiento de movimiento (Awasthi \& Joshi, 2015) (Gouwanda \& Senanayake, 2011) (Najabi, Aminian, Loew, Blanc, \& Robert, 2002). Por otro lado, un acelerómetro es un dispositivo electromecánico que mide las fuerzas de aceleración, ya sean estáticas o dinámica. 
Se conoce que la posición puede ser obtenida a través de la doble integración de la señal de aceleración; sin embargo, este simple procedimiento conduce a errores que crecen en el tiempo debido al propio proceso de la integración. Para la estimación de la posición es necesario la estimación de la orientación para poder transformar de un sistema coordenado a otro. Dicha estimación es usada para determinar las componentes de aceleración lineal o de velocidad lineal en los ejes de movimiento (Vela Pena, 2016). La orientación del IMU en 3D (y por tanto del cuerpo que está unido a él) puede ser obtenida a través de la señal del giroscopio (Pasciuto, Ligorio, Bergamini, \& Vannozzi, 2015). El proceso de integración de la señal del giroscopio también conduce a errores que crecen a lo largo del tiempo; además, las condiciones iniciales para la integración a menudo necesitan ser determinadas.

La utilización de forma independiente de las señales de aceleración lineal obtenida del acelerómetro y de las señales de velocidad angular brindadas por el giroscopio, no es recomendable debido a los errores que cada una de forma independiente puede presentar (Pasciuto, Ligorio, Bergamini, \& Vannozzi, 2015) (Pedley, 2013) (Varesano, 2011). Por esta razón es necesario que al utilizar la información que ambos brindan se emplee un algoritmo que compense la debilidad de cada sensor, y se obtenga un resultado más preciso. Para lograr esto muchos investigadores proponen implementar la fusión de sensores (Abyarjoo, Barreto, Cofino, \& Ortega, 2015) (Luinge \& Veltink, 2005) (Kam, Zhu, \& Kalata, 1997); sin embargo, no es posible realizar la fusión de sensores si no existe otro observador externo al IMU y si el dispositivo no está sometido a ningún movimiento lineal.

La aplicación de sensores inerciales para el monitoreo de la marcha humana es ampliamente utilizada a nivel mundial y que aún la estimación de la posición a través de IMUs constituye un tema abierto de investigación. Por su naturaleza los IMUs requieren, por un lado, de "observadores" extras (encarecedores del sistema) que permitan implementar procedimientos de estimación posición precisos como, por ejemplo: GPS, sensores de presión, sensores ópticos y cámaras de video. Por otro lado, todos los intentos dirigidos a implementar la estimación de la posición sin el uso de tales observadores, aplicando sobre todo fusión de sensores, siguen siendo infructuosos. Solo, asumiendo restricciones en la aplicación, se podrá alcanzar mejores resultados. De aquí surge la necesidad de desarrollar algoritmos matemáticos capaces de estimar variables espacio-temporales con mayor precisión, teniendo en cuenta características particulares de las pruebas.

\section{ALGORITMO DE ESTIMACIÓN DE POSICIÓN EN SISTEMAS DE TRANSPORTACIÓN}

En (Hemminki, Nurmi, \& Tarkoma, Diciembre 2014) se estima la posición en una aplicación de dispositivos móviles. El algoritmo se basa en la detección de periodos de estabilidad en una aplicación de estimación de ruta en autobuses. La investigación desarrollada en (Hemminki, Nurmi, \& Tarkoma, Diciembre 2014) contribuye a la obtención de un enfoque novedoso para la estimación de la gravedad y la aceleración lineal. El mismo funciona con una combinación de mediciones de acelerómetros y giroscopios. El algoritmo consiste en detectar los llamados puntos claves que corresponden a mínimos locales en la señal de aceleración, a través de un trabajo realizado con ventanas. Para cada punto clave encontrado, se asigna un valor de estabilidad que refleja la variabilidad general de las mediciones alrededor del punto clave detectado. A partir de los puntos claves obtenidos se realiza una estimación de la componente de gravedad en las ventanas que contienen puntos no claves, interpolando entre los puntos claves detectados. Como algunos de los puntos claves detectados pudieran ser erróneos se procede a realizar una validación de los mismos antes de que se interpolen las estimaciones de gravedad. El algoritmo controla los cambios de orientación y reinicia el proceso de estimación de gravedad cada vez que se detecte un cambio sustancial. Al estimarse la componente de gravedad se rotan las mediciones del acelerómetro eliminadas por la gravedad en el plano de referencia global para tener una estimación de la aceleración lineal general. Los cambios relativos del dispositivo y la aceleración horizontal son empleados para obtener las componentes laterales y longitudinales de la aceleración. Este algoritmo, de forma general, es dividido en el cálculo de la estabilidad, detección de los puntos clave y validación de los mismos, obtención de posibles cambios en la orientación, estimación de la gravedad y finalmente la extracción de la aceleración lineal para obtener la posición.

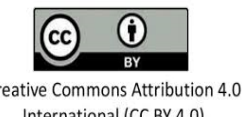




\section{ORANGE JOURNAL}

\section{Metodología}

Propuesta de algoritmo para estimar tamaño de zancada basado en la estimación de posición en un sistema de transportación

Durante el proceso de marcha humana, cuando el sensor se encuentra ubicado en el pie (ver figura 1), ocurren períodos de estabilidad. Por esta razón, el algoritmo de (Hemminki, Nurmi, \& Tarkoma, Diciembre 2014) pudiera ser adaptado a las condiciones de una aplicación de marcha; en esto consiste el trabajo de investigación presentado. En la propuesta de algoritmo no se realiza la validación de los puntos claves y no se atienden los cambios en la orientación, pues se ha considerado que la marcha se realizará de la forma más recta posible; como la marcha se realizará en un lugar específico, tampoco se tendrán en cuenta los cambios en la orientación.

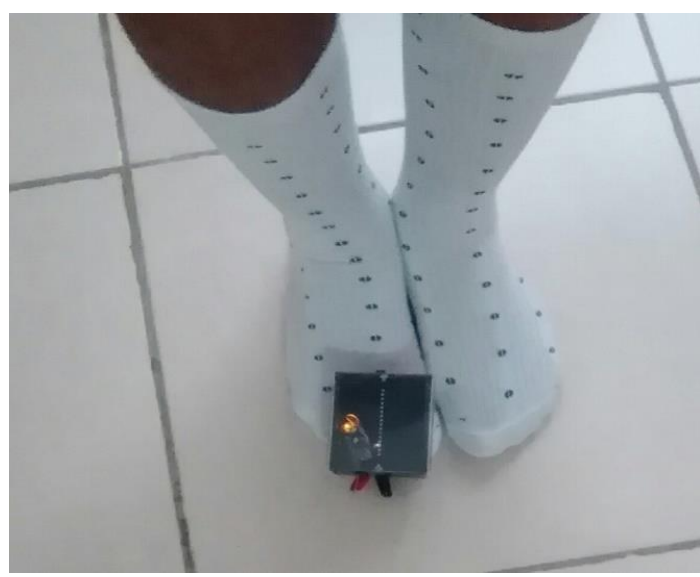

Figura 1. Ubicación del sensor en la parte delantera del pie.

Cálculo de la estabilidad (elaborada por el autor)

El primer paso de la implementación del algoritmo es la detección de los puntos claves, y para ello se calcula la estabilidad de la marcha reflejada en las señales de los acelerómetros y de los giroscopios. Los periodos donde se debe encontrar estabilidad se debe corresponder con aquellos momentos donde el pie que tiene colocado el sensor, esté en la fase de reposo, pues hay ausencia de movimiento, como se muestra en la figura 2.

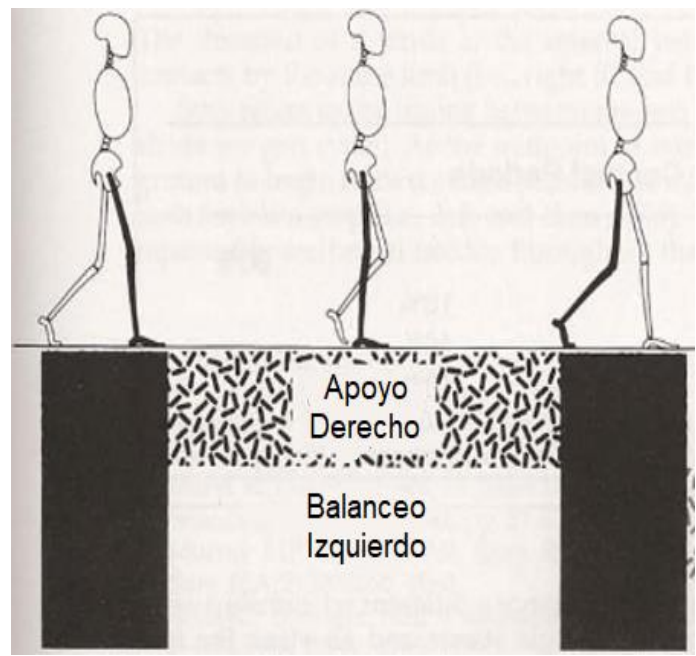

Figura 2. Fase de apoyo del pie derecho durante un ciclo de marcha (modificada por el autor tomada de (Perry, 1992)) 
Para mejorar la robustez de la detección de puntos claves, se combinan mediciones de acelerómetros y giroscopios para asignar un puntaje de estabilidad que refleje la variabilidad general de las mediciones dentro de una ventana específica. Solo las ventanas con una estabilidad suficientemente alta se consideran puntos claves. El cálculo de la estabilidad es representado en la siguiente expresión:

$$
S(X)=\alpha_{\sigma} \sigma_{X}+\alpha_{\mu} \Delta \mu_{X}+\alpha_{\omega} \omega_{X}
$$

donde $\alpha_{\sigma}, \alpha_{\mu}$ y $\alpha_{\omega}$ son términos de peso; $\sigma_{X}$ representa la desviación estándar de las mediciones; $\Delta \mu_{X}$ es la diferencia entre el valor medio entre las ventanas de la medición actual y la medición anterior; y $\omega_{X}$ es el valor medio de la velocidad angular de cada ventana. La ecuación (1) refleja que el cálculo de la estabilidad es una combinación de acelerómetros y de giroscopios.

Detección de los puntos clave

Para identificar los mejores puntos claves que pudieran existir, se compara la estabilidad de una ventana de medición con un umbral empírico predefinido. El algoritmo para detectar los puntos claves se muestra a continuación:

$$
\begin{gathered}
\text { 1: } S_{i}=\text { Estabilidad }(i) \\
\text { 2: } \operatorname{Si~} S_{i} \leq E_{s} \\
\text { 3: } K_{i}^{S}=S_{i} \\
\text { 4: } K_{i}^{G}=\text { mean }(\text { Accel })
\end{gathered}
$$

El primer paso en la detección de los puntos claves es la asignación a una variable llamada $S_{i}$ del valor de estabilidad calculado en la ecuación (1); esto se realiza en la línea 1 del algoritmo. La línea 2 del algoritmo indica que si el valor de estabilidad de la ventana es mayor que el umbral $E_{s}$, entonces en esa ventana habrá un punto clave que tomará el valor de la estabilidad, como es indicado en la línea 3; además, de cumplirse la condición, también se obtendrán los valores de gravedad en las mismas ventanas donde se guardará el valor medio de las señales de los acelerómetros, línea 4.

Estimación de la gravedad

Para el caso de los puntos no claves, la estimación de la gravedad se realiza a partir de las gravedades obtenidas en el punto clave anterior $(K a)$ y el punto clave posterior $(K b)$ a dichos puntos no claves. La estimación de la gravedad $G(x)$ de los puntos no claves se realiza a través de la siguiente expresión:

$$
G(x)=(1-\alpha) G(x-1) * w
$$

donde $\alpha$ es un término de peso de valor predeterminado en función de los mejores resultados que arrojasen los experimentos, $G(x-1)$ es el valor de gravedad del último punto clave detectado $K a$; y $w$ es la matriz de rotación derivada de las mediciones del giroscopio.

Para realizar la estimación de gravedad se utiliza un esquema de interpolación bidireccional para ajustar la gravedad estimada. El uso de un esquema bidireccional ayuda a mitigar la deriva al reducir la distancia entre valores interpolados y estimaciones de gravedad. Se obtienen dos estimaciones de gravedad utilizando las secuencias $G a=G(x 1 \ldots n)$ como (interpolación hacia adelante) y $G b=G(x n \ldots 1)$ (interpolación hacia atrás).

La estimación de la gravedad final $G$, está dada por la siguiente expresión:

$$
G=(1-W) G a(x 1 \ldots n)+W G b(x n \ldots 1)
$$




\section{ORANGE JOURNAL}

donde la función de peso no lineal, W, está dada por la siguiente expresión:

$$
\left\{\begin{array}{c}
W=2(x / n)^{2}, 1 \leq i-1<c \\
W=1-2\left(\frac{x-n}{n}\right)^{2}, c \leq i-1<n
\end{array}\right.
$$

donde $n$ es la cantidad de puntos no claves más uno, $i$ toma valores desde 1 hasta $n$, donde 1 corresponde al punto clave que le antecede a un punto no clave, $n$ es el punto clave que le precede a un punto no clave, $x=i-1$ y $c$ es un parámetro definido en (Hemminki, Nurmi, \& Tarkoma, Diciembre 2014). Puede notarse que $W=0$ y $W=1$ corresponden a los puntos claves, a los cuales no hay que realizarles estimación de gravedad final, por tanto no se tendrán en cuenta en la función de peso a emplear en la propuesta.

Se puede notar que para la estimación de la gravedad final, como se muestra en la ecuación (3) es determinante la función de peso no lineal determinada por la ecuación (4) en dependencia del valor de $i$. La ecuación (4) difiere de la correspondiente en (Hemminki, Nurmi, \& Tarkoma, Diciembre 2014), para poder resolver problemas en la definición de la ecuación en (Hemminki, Nurmi, \& Tarkoma, Diciembre 2014).

Estimación de la aceleración lineal y de la posición

Una vez que se ha estimado la componente de gravedad, se descompone la aceleración lineal en un vector tridimensional consistente en aceleraciones vertical $L_{v}$, longitudinal $L l_{o}$ y lateral $L l_{a}$. Estos vectores se conforman rotando las medidas de los acelerómetros en el marco de referencia a partir de la matriz de rotación obtenida de las estimaciones de gravedad como se muestra en la siguiente expresión:

$$
A=\left\{\begin{array}{l}
L_{v}=A(1,:) * w 1 \\
L l_{o}=A(2,:) * w 1 \\
L l_{a}=A(3,:) * w 1
\end{array}\right.
$$

donde $A(1,:), A(2,:)$ y $A(3,:)$ son las descomposiciones de la matriz de aceleración y $w 1$ es la matriz de rotación obtenida de las estimaciones de gravedad.

Una vez obtenidos los vectores vertical, lateral y longitudinal que conforman la aceleración lineal, se procede a realizar la doble integración para obtener la posición como se muestra a continuación:

$$
\operatorname{Posición}(x)=\iint L_{v}
$$

La aplicación del algoritmo fue validada mediante el empleo de señales simuladas y posteriormente empleando señales reales. Las señales simuladas que un inicio tendrán condiciones ideales posteriormente se les incorporará ruido a las señales correspondientes a los acelerómetros y deriva a las señales que corresponden a los giroscopios, para acercarlas a condiciones reales. La incorporación de estos fenómenos a las señales permitirá realizar una valoración de cómo se verá afectada el valor de amplitud de la señal de posición cuando no existan las condiciones ideales. La efectividad del algoritmo propuesto será comprobada con el valor medio de la diferencia entre la señal de posición esperada y la señal de posición estimada.

Como se describió en la sección 3 el algoritmo de estimación de posición propuesto en este trabajo se basa en el trabajo con ventanas que es el resultado de realizar divisiones de la señal. En este trabajo las ventanas se fijaron en un tamaño de 10 muestras. El ciclo de la marcha es considerado como una simple secuencia desde que un mismo pie tiene contacto inicial con el suelo y posteriormente vuelve a tener contacto final con este (Perry, 1992), como se muestra en la figura 2. Este ciclo es dividido en una fase de apoyo y otra de balanceo, en la que cada una ocupa el $60 \%$ y el $40 \%$, respectivamente (Perry, 1992). La zancada, uno de los elementos de la marcha, es equivalente a un ciclo de marcha. La duración de una zancada debe ser aproximadamente $2 \mathrm{~s}$, lo cual refleja que la fase de balanceo tendría una duración de aproximadamente 0.8 s. Como la frecuencia de muestreo aplicada por el sistema electrónico es de $50 \mathrm{~Hz}$, esto significaba que para la fase de balanceo se tendrían aproximadamente 40 muestras. Con el objetivo de que en esta fase de 
la marcha hubiese la mayor cantidad posible de ventanas, con la mayor cantidad posible de muestras, para detectar la mayor cantidad de cambios durante el movimiento, se decidió utilizar 10 muestras por ventanas. La utilización de ventanas más pequeñas permitiría obtener una mayor cantidad de información instantánea, pero se obtendrían menos muestras para realizar el promediado, lo cual implicaría una menos precisa detección de puntos claves y a su vez, una imprecisa estimación de la gravedad.

Como las señales de aceleración con las que se trabajó tienen un tamaño de 248 muestras (equivalente a un registro de marcha de aproximadamente $5 \mathrm{~s}$ ), el total de ventanas con las que se trabajó fue de 24. Cada una de las ventanas obtenidas estuvo caracterizada por los diferentes promedios que se realizaron a las 10 muestras contenidas en cada una de ellas. Luego, la "señal" resultante de la aplicación del algoritmo propuesto, tuvo 24 muestras. Por tanto, para mostrar la señal estimada con respecto a la señal esperada en una misma gráfica, ambas debían tener la misma cantidad de muestras. Es por eso que la señal de posición esperada también fue tratada con un proceso de promediado (ventana de tamaño 10, sin solapamiento), dando como resultado una señal de posición de tamaño de 24 muestras.

Como la aplicación del algoritmo propuesto está dada en la estimación de la posición en una aplicación de marcha humana (determinación de tamaño de zancada), solo se visualizará la señal de posición resultante del eje $x$, que es la que estará relacionada con el parámetro de la marcha humana a estimar.

\section{Resultados y discusión}

\section{Trabajo con señales simuladas}

Para la validación de la propuesta de algoritmo se simuló un recorrido en el espacio, por tanto, con determinados valores de aceleración en los 3 ejes, sin variación en la inclinación del IMU. La descripción de la señal de posición fue la siguiente:

La componente en el eje $x$ de la señal de posición tiene un comportamiento constante igual a 0 en un tiempo de $0 \mathrm{~s}$ a $1 \mathrm{~s}$, de $1 \mathrm{~s}$ a $2 \mathrm{~s}$ tiene un comportamiento cúbico positivo, de $2 \mathrm{~s}$ a $3 \mathrm{~s}$ mantiene un amplitud constante igual a uno, de $3 \mathrm{~s}$ a $4 \mathrm{~s}$ tiene otro comportamiento cúbico positivo y de $4 \mathrm{~s}$ a $5 \mathrm{~s}$ se mantiene constante con amplitud igual a 2. La señal de posición en el eje $y$, desde $0 \mathrm{~s}$ a $5 \mathrm{~s}$ se mantiene en 0 ; en el eje $z$ esta señal de posición tiene un comportamiento constante igual a 0 en un tiempo de $0 \mathrm{~s}$ a $1 \mathrm{~s}$, de $1 \mathrm{~s} \mathrm{a}$ $2 \mathrm{~s}$ su comportamiento es cúbico positivo, de $2 \mathrm{~s}$ a $3 \mathrm{~s}$ mantiene un amplitud constante igual a 1, de $3 \mathrm{~s}$ a 4 s tiene un comportamiento cúbico negativo y de $4 \mathrm{~s}$ a $5 \mathrm{~s}$ se mantiene constante igual a 0 . La señal de ángulos de inclinación tanto en los ejes $x, y$ y $z$ tiene una amplitud constante igual a 0 desde $1 \mathrm{~s}$ a $5 \mathrm{~s}$.

La doble derivación de la señal de posición devuelve señales de aceleración, esto puede observarse en la figura 3, dichas señales son correspondientes a las que devuelve los acelerómetros. La derivación de la señal de ángulos de inclinación devuelve señales de velocidad angular con valor igual a 0, dichas señales corresponden, por su parte, a las que devuelve un giroscopio.

En las figuras 3 y 4 se muestran en las señales de posición generadas en los tres ejes coordenados y en las señales de aceleración en los tres ejes coordenados (obtenidas al aplicar la doble integración), respectivamente. 


\section{ORANGE JOURNAL}

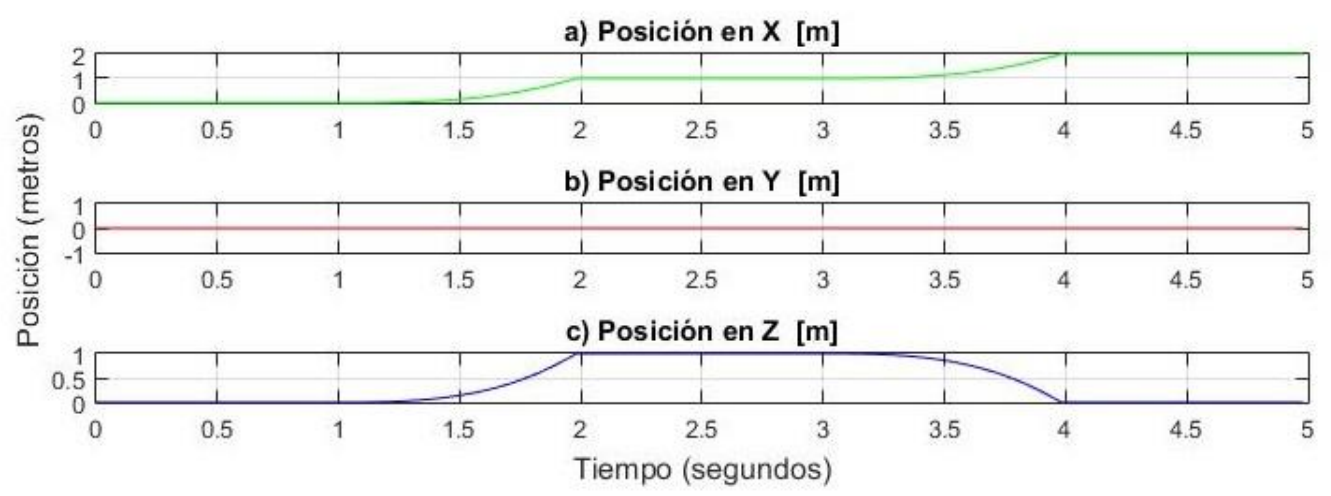

Figura 3. Señal de posición generada (elaborada por el autor)

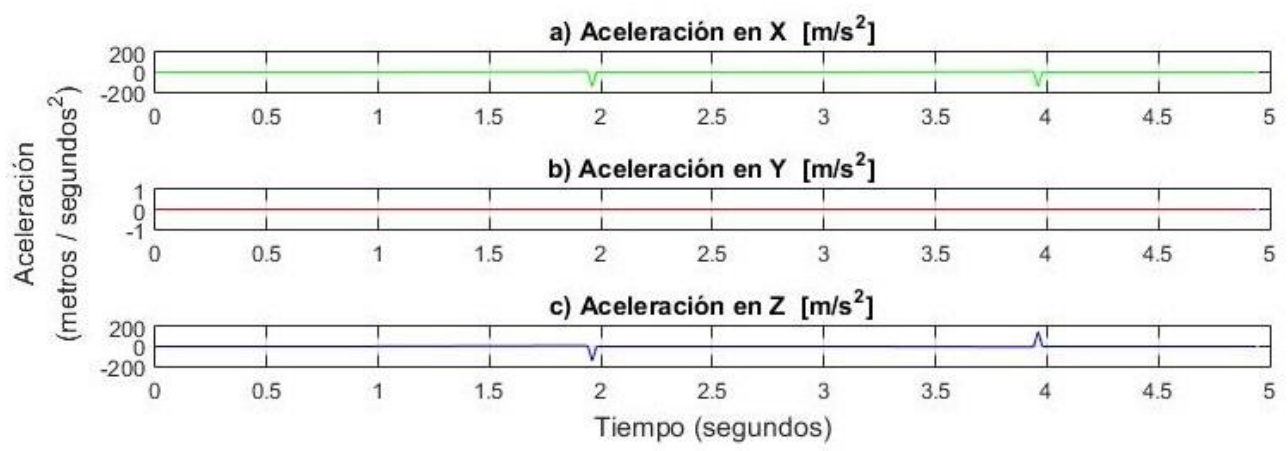

Figura 4. Señal de aceleración obtenida con la integración (elaborada por el autor)

$\mathrm{Al}$ aplicarse el algoritmo propuesto a la señal de aceleración, se obtuvo la señal de posición, utilizando para ello los valores de peso de la función de estabilidad donde $\alpha_{\sigma}=0.1, \alpha_{\mu}=0.45$ y $\alpha_{\omega}=0.45$, como se recomendó en (Hemminki, Nurmi, \& Tarkoma, Diciembre 2014) y $E_{s}=1$. En la figura 5 , se representan la posición esperada (línea verde) y la posición estimada (línea roja). En esta figura se puede observar la diferencia existente entre estas señales de posición. La diferencia entre el valor final de ambas señales es de $0.32 \mathrm{~m}$ y el valor medio de la diferencia entre estas señales es de $0.06 \mathrm{~m}$.

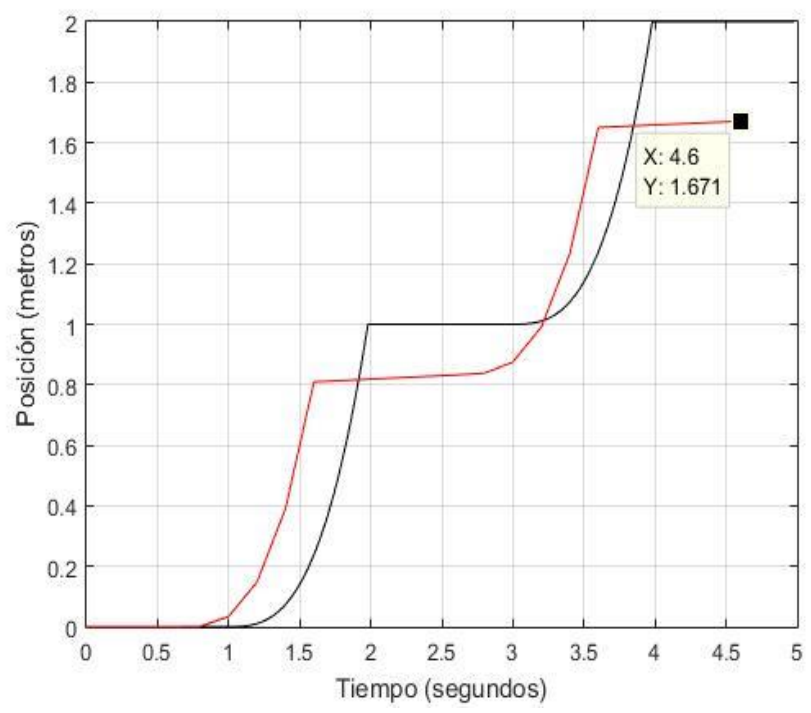

Figura 5. Señal de posición esperada (negro) vs Señal de posición estimada (rojo) (elaborada por el autor) 
Con el objetivo de analizar el efecto del ruido en la señal de los acelerómetros en el resultado del algoritmo propuesto, a las señales simuladas correspondientes a los acelerómetros se les sumó ruido con distribución normal, media cero y desviación típica de $0.06 \mathrm{~m} / \mathrm{s} 2$. Para el algoritmo propuesto se mantienen los mismos parámetros empleados en el primer experimento. En la figura 6 se muestran la posición esperada (línea negra) y la posición estimada (línea roja). En esta figura se puede observar con claridad la diferencia existente entre estas señales de posición. La diferencia entre el valor final de ambas señales es de $0.49 \mathrm{~m}$ y el valor medio de la diferencia de estas señales es de $0.14 \mathrm{~m}$.

Si se compara con el resultado previo obtenido, se puede notar que la diferencia promedio entre las señales aumentó, lo cual indica que el ruido en la señal de los acelerómetros afecta la efectividad del algoritmo propuesto.

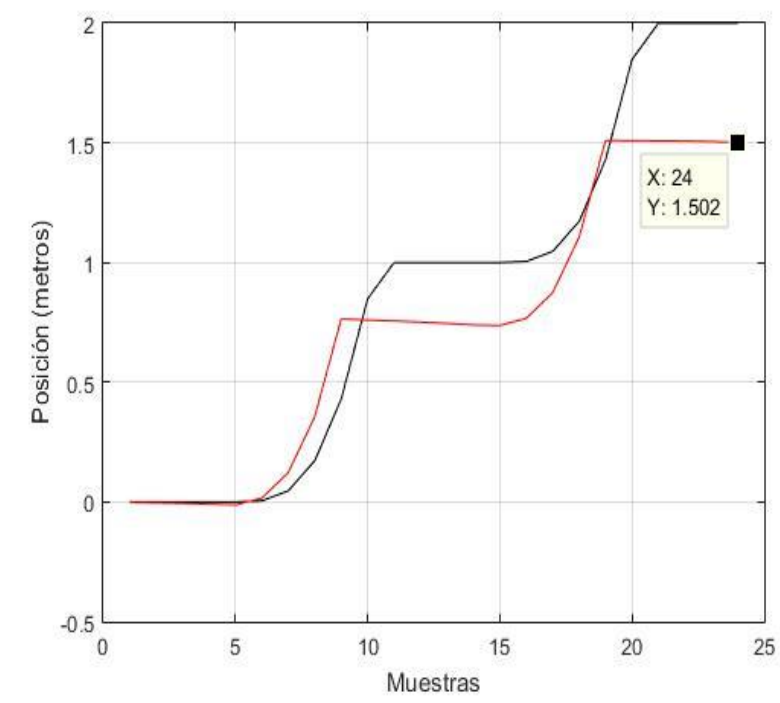

Figura 6. Señal de posición esperada (negro) vs Señal de posición estimada (rojo) (ruido en señal de acelerómetro) (elaborada por el autor

Con el objetivo de analizar el efecto de la deriva en las señales de los giroscopios en el resultado del algoritmo propuesto, a las señales simuladas correspondientes a los giroscopios se les sumó una constante igual a 0.1 Para el algoritmo propuesto se mantienen los mismos parámetros empleados en el primer experimento. En la figura 7 se muestran la posición esperada (línea negra) y la posición estimada (línea roja). En esta figura se puede observar que también ocurre una disminución en el valor de la señal de posición estimada en comparación con la señal de posición esperada. La diferencia entre el valor final de ambas señales es de $0.47 \mathrm{~m}$ y el valor medio de la diferencia de estas señales es de 0.12 . Si se compara este resultado con el resultado del primer experimento ( promedio entre las señales aumentó, lo cual indica que la deriva en la señal de los giroscopios también afecta la efectividad del algoritmo propuesto. 


\section{ORANGE JOURNAL}

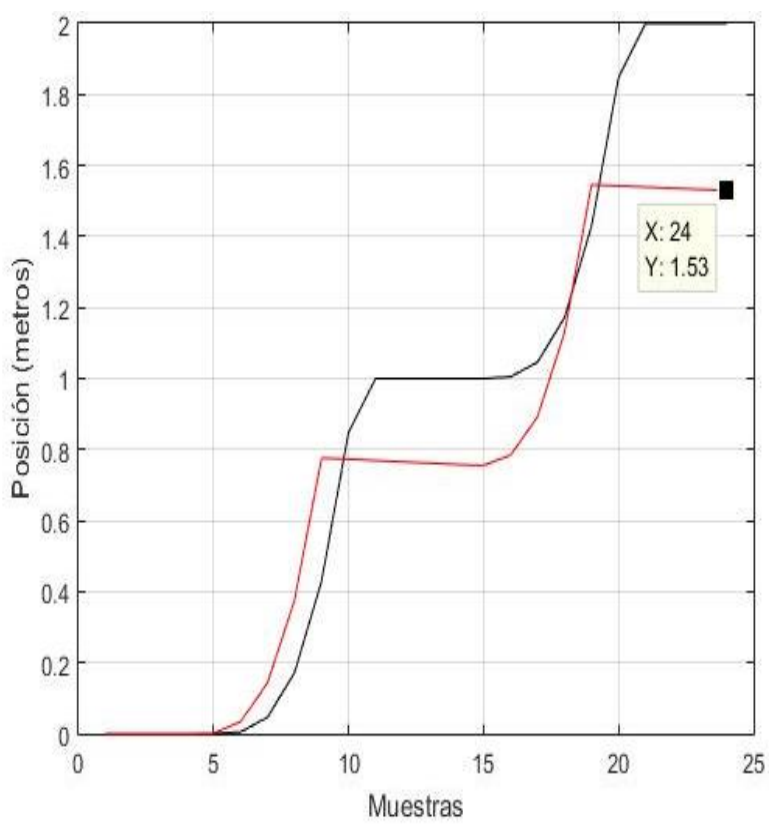

Figura 7. Señal de posición esperada (negro) vs Señal de posición estimada (rojo) (deriva en el giroscopio) (elaborada por el autor)

Con el objetivo de acercar la efectividad del experimento propuesto al caso real: ruido en la señal de los acelerómetros y deriva en la señal de los acelerómetros, se trabajó con las mismas condiciones establecidas en el segundo y tercer experimentos, al mismo tiempo. Bajo estas condiciones, los valores de los pesos de la función de estabilidad del algoritmo se fijaron como sigue: $\alpha_{\sigma}=0.2, \alpha_{\mu}=0.4$ y $\alpha_{\omega}=0.4$, buscando una mayor efectividad en el trabajo con dicha función (mayor efectividad en la detección de los puntos clave); sin embargo el valor de $E_{S}$ no fue necesario variarlo. La señal de posición esperada y la señal de posición estimada con el algoritmo propuesto, en presencia de ruido y de deriva, se muestran en la figura 8.

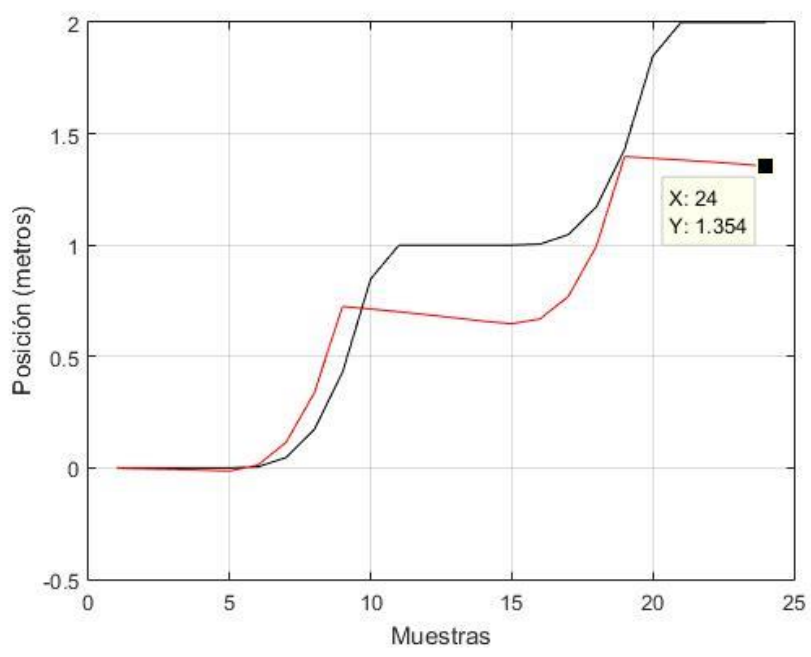

Figura 8. Señal de posición esperada (negro) vs Señal de posición estimada (rojo) en presencia de ruido y de deriva (elaborada por el autor)

Se puede observar en la figura 8 que la estimación de la señal de posición bajo las condiciones de presencia de ruido en las señales de acelerómetros y de deriva en las señales de giroscopios se deteriora más que en resto de los experimentos. La diferencia existente entre la señal de posición esperada y la señal que sí 
presenta ruido y deriva es de $0.64 \mathrm{~m}$, mientras que el valor medio de la diferencia de estas señales es de 0.32 .

\section{Trabajo con señales reales}

El trabajo con las señales reales fue realizado utilizando las señales obtenidas del sistema electrónico ya diseñado, basado en IMU, para aplicar al análisis de la marcha.

Para la obtención de las señales el IMU fue ubicado en la puntera del pie (ver figura 1). La implementación del algoritmo es realizada empleando los mismos parámetros utilizados durante el trabajo con señales simuladas cuando había presencia de ruido y de deriva. La aplicación del algoritmo fue realizado utilizando una frecuencia de muestreo igual a $50 \mathrm{~Hz}$ y una cantidad de 10 muestras por ventanas.

El ruido de las señales de aceleración fue disminuido aplicando filtrado paso bajo con corte en los $15 \mathrm{~Hz}$, atendiendo a que el contenido espectral fundamental de la marcha humana, en las señales de aceleración, se concentra por debajo de los $5 \mathrm{~Hz}$. La deriva de las señales de los giroscopios se redujo también al substraer, a dichas señales, el valor medio de las mismas, correspondiente al primer segundo (en el cual aún no se ha iniciado la marcha).

Para la estimación de la posición se realizaron dos experimentos: (1) realización de un paso alcanzando una posición final igual a $0.70 \mathrm{~m}, \mathrm{y}$ (2) realización de dos pasos con valor de posición parcial de $0.50 \mathrm{~m}$ y final de $1.20 \mathrm{~m}$.

Al aplicarse el algoritmo propuesto, se obtendrá una señal de posición estimada, la cual será comparada con una señal de posición esperada. Dicha señal de posición esperada es obtenida a través de los instantes de estabilidad de la señal de la marcha obtenidos con el IMU, que son los instantes en donde el pie que tiene ubicado el sensor se encuentra en la fase de apoyo. La efectividad del algoritmo, empleando señales reales, fue comprobada a través del vector de diferencia entre la posición final esperada y la posición final estimada.

Al aplicarse el algoritmo propuesto a la primera señal real, la posición estimada se muestra en la figura 9. En esta figura se puede ver que se estima un valor de posición final igual a $0.65 \mathrm{~m}$ (en rojo), siendo la posición final esperada igual a $0.70 \mathrm{~m}$ (en azul). En este caso el valor medio de la diferencia de la señal esperada con la señal estimada es de $0.05 \mathrm{~m}$.

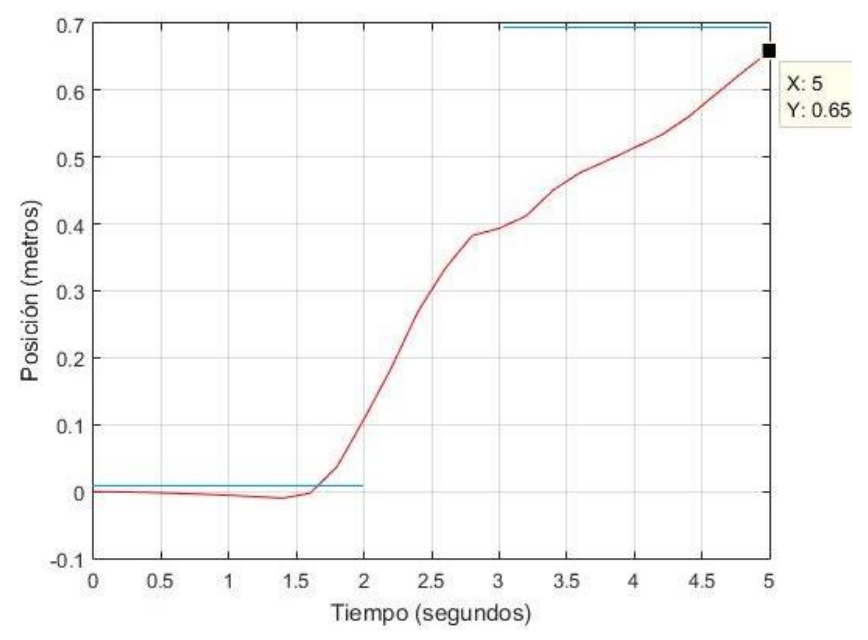

Figura 9. Señal de posición estimada en el eje $x$ en el experimento 1 (elaborada por el autor)

En la figura 9 también se puede observar que hay unas líneas de color azul que corresponden a los instantes en los que el pie del sensor está en apoyo. 


\section{ORANGE JOURNAL}

Al aplicar el algoritmo a la segunda señal real, se obtiene la señal de posición estimada mostrada en la figura 10. En esta figura se puede ver que se estima un valor de posición igual a $1.12 \mathrm{~m}$ siendo la posición esperada igual a $1.20 \mathrm{~m}$. En este caso el valor medio de la diferencia de la señal esperada con la señal estimada es de $0.08 \mathrm{~m}$.

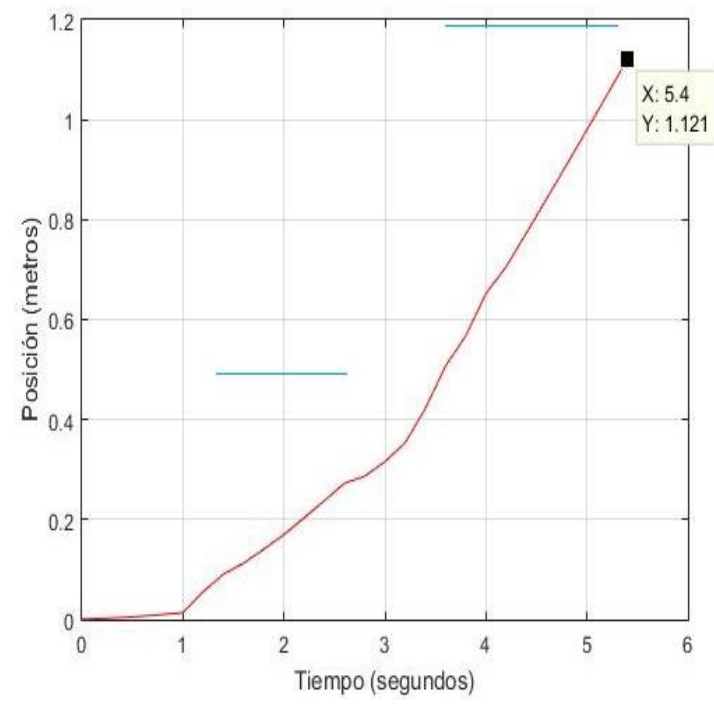

Figura 10. Señal de posición estimada en el eje $x$ en el experimento 2 (elaborada por el autor)

En la figura 10, la línea azul de $0 \mathrm{~s}$ a $1.2 \mathrm{~s}$ indica que durante ese tiempo el pie que tiene fijado el sensor estuvo apoyado sobre el suelo. La otra línea azul de $2.3 \mathrm{~s}$ a cerca de $3.4 \mathrm{~s}$, se corresponde con un nuevo apoyo de ese pie con una posición parcial igual a $0.5 \mathrm{~m}$. La última línea azul de $4.5 \mathrm{~s}$ a cerca de $5.6 \mathrm{~s}$, se corresponde con un nuevo apoyo de ese pie, en la posición final de $1.12 \mathrm{~m}$. Además, se puede observar, que a pesar de que en el primer paso incurre en un error más apreciable, en el segundo paso, tiene una mayor efectividad

\section{Conclusiones}

El objetivo fundamental de la investigación fue cumplido pues se logró con cierto grado de efectividad, la estimación de la posición, que, para la forma en que se colocó el sensor, en una aplicación de marcha, corresponde con la longitud de la zancada. La validación del algoritmo propuesto con señales simuladas en diferentes ambientes permitió llegar a conclusiones que resultaron imprescindibles durante el trabajo con las señales reales provenientes de acelerómetros y giroscopios.

A pesar de haberse aplicado métodos de reducción de ruido en el acelerómetro y de reducción de la deriva en el giroscopio, estos fenómenos siempre resultaron determinantes para llegar a una efectividad mayor en la estimación de la posición.

El empleo de una frecuencia de muestreo de $50 \mathrm{~Hz}$, relativamente baja para la aplicación del algoritmo propuesto, implicó que se utilizaran ventanas de gran tamaño. Aunque la tendencia en las investigaciones alrededor del tema abordado es la utilización de frecuencias de muestreo bajas, se recomienda la utilización de frecuencias mayores, que permitirían utilizar mayor número de ventanas con una mayor cantidad de muestras.

\section{Agradecimientos}

La investigación que da origen a los resultados presentados en la presente publicación recibió fondos de la Oficina de Gestión de Fondos y Proyectos Internacionales bajo el código PN305LH13-050". 


\section{Referencias Bibliográficas}

Abyarjoo, F., Barreto, A., Cofino, J., \& Ortega, F. R. (2015). Implementing a sensor fusion algorithm for $3 \mathrm{D}$ orientation detection with inertial/magnetic sensors. In Innovations and advances in computing, informatics, systems sciences, networking and engineering (págs. 305-310). Cham: Springer, doi:10.1007/978-3319-0677-5_4/

Awasthi, S., \& Joshi, A. (febrero, 2015). MEMS accelerometer based system for motion analysis. 2nd International Conference on Electronics and Communication Systems (ICECS), (págs. 762-767). Coimbatore, India.

Chen, K., \& Basset, D. J. (2005). The technology of accelerometry-based activity monitors: current and future. Med Sei Sports Exere, 37(11), 490-500.

Corder, K., Ekelund, U., Steele, R. M., Wareham, N. J., \& Brage, S. (2008). Assessment of physical activity in youth. Journal of Applied Physiology, 105(3), 977-987.

Garcia, D., \& Bueno, C. (2010). Desempeño Físico en adultos mayores sanos, del municipio Plaza de la Revolución. GeroInfo, 5(1).

Garcia, D., Morejon, S., De Dios, C., \& Rodriguez, Z. (2018). Las pruebas de desempeño físico en el pronóstico de desenlaces adversos en los ancianos. MediSan, 22(6), 466-470.

Garcia, D., PIinera, J. A., Garcia., \& Bueno, C. (2020). Estudio de la fuerza de agarre en adultos mayores del municipio Plaza de la Revolución. Revista Cubana Médica Deporte Cultura Física, 8(1), 1-13.

Gerety, M., Mulrow, C., Tuley, M., Hazuda, H., Lichetensein, M., \& Bohannon, R. (1993). Development and validation of a physical performance instrument for the functionally impaired elderly: The Physical Disability Index (PDI). Journal of Gerontology Series A: Biomedical Sciences and Medical Sciences, 48(2), 33-38.

Gouwanda, D., \& Senanayake, S. (2011). Periodical gait asymmetry assessment using real-time wireless gyroscopes gait monitoring system. J. Med. Eng. Technol., 35(8), 432- 440.

Hannik, J., Kautz, T., Pasluosta, C. F., Barth, J., Schulein, S., Gabmann, K., \& Eskofier, B. (2017). Mobile stride length estimation with deep convolutional neural networks. IEEE journal of biomedical and health informatics, 22(2), 354-362.

Hemminki, S., Nurmi, P., \& Tarkoma, S. (diciembre, 2014). Gravity and linear acceleration estimation on mobile devices. In Proceedings of the 11th International Conference on Mobile and Ubiquitous Systems: Computing, Networking and Services, (págs. 50-59). London.

Kam, M., Zhu, X., \& Kalata, P. (1997). Sensor fusion for mobile robot navigation. Proceedings of the IEEE, 85(1), 108-119.

Kavanagh, JJ, \& Menz, H. B. (2008). Accelerometry: a technique for quantifying movement pattems dudng walking. Gait Posture, 28(1), 1-15.

Lee, C. Y., \& Lee, J. J. (2002). Estimation of walking behavior using accelerometers in gait rehabilitation. International Journal of Human-friendly Welfare Robotic Systems, 3(2), 32-36.

Loredo Medina, R. (2011). Sistema de monitoreo de la marcha como apoyo al cuidado de adultos mayores. (Tesis de Maestría), Centro de Investigación Científica y de Educación Superior de Ensenada, Baja California. Recuperado el 19 de agosto de 2019, de https://cicese.repositorioinstitucional.mx/jspui/handle/1007/1547

Luinge, H. J., \& Veltink, P. H. (2005). Measuring orientation of human body segments using miniature gyroscopes and accelerometers. Medical and Biological Engineering and computing, 43(2), 273-292.

Martin, E. (2011). Novel method for stride length estimation with body area network accelerometers. In 2011 IEEE Topical Conference on Biomedical Wireless Technologies, Networks, and Sensing Systems, (págs. 79-82).

Menendez Alvarez, J. (2018). Desarrollo de un sistema electrónico experimental para la medición de variables físicas necesarias en la determinación de parámetros de marcha en ancianos. (Tesis en opción al título de ingeniero en Telecomunicaciones y Electrónica) Universidad Tecnológica De La Habana José Antonio Echeverría CUJAE. Cuba. Obtenido de https://scholar.google.es/scholar?cluster=1575214438424323267\&hl=es\&as_sdt=2005\&sciodt= 0,5

Najabi, B., Aminian, K., Loew, F., Blanc, Y., \& Robert, P. A. (2002). Measurement of stand-sit and sitstand transitions using a miniature gyroscope and its application in fall risk evaluation in the elderly. IEEE Trans. Biomed. Eng., 49(8), 843-851. 


\section{ORANGE JOURNAL}

Pang, J., \& Sing, I. (Octubre 2011). Accelerometer Based Real-Time Remote Detection and Monitoring of Hand Motion. Proceedings of the World Congress of Engineering and Computer Science, (págs. 19-21). San Francisco, USA.

Pasciuto, I., Ligorio, G., Bergamini, E., \& Vannozzi, G. (2015). How Angular Velocity Features and Different Gyroscope Noise Types Interact and Determine Orientation Estimation Accuracy. Sensors, 15(9), 23983-24001.

Pedley, M. (2013). Tilt sensing using a three-axis accelerometer. Freescale semiconductor application note, 1, 1, 2012-2013.

Perry, J. (1992). Gait Analysis Normal and Pathological Function. USA: SLACK.

Peruzzi, A., Della, U., \& Cereatti, A. (2011). Estimation of stride length in level walking using an inertial measurement unit attached to the foot: A validation of the zero velocity assumption during stance. Journal of Biomechanics, 44(10), 1991-1994.

Rantanen, T., Guralnik, J., Ferrucci, L., \& Leveille, S. (1999). Coimpairments: strength and balance as predictors of severe walking disability. Journals of Gerontology Series A: Biomedical Sciences and Medical Sciences, 54(4), 172-176.

Sijobert, B., M., B., J., D., Pissard, R., Geny, C., \& Coste, C. A. (2015). Implementation and validation of a stride length estimation algorithm, using a single basic inertial sensor on healthy subjects and patients suffering from Parinson's disease. Electronic Healthcare, 7, 704-714. doi: $10.4236 /$ health 2015.76084

Vara Rodriguez, D. (2014). Sistemas para determinar la posición y orientación de herramientas quirúrgicas en operaciones de cirugía laparoscópica. (Tesis en opción al título de Ingeniería Electrónica Industrial y Automática) Escuela de Ingeniería de Industriales, Departamento de Ingeniería de Sistemay Automática, Mexico.

Varesano, F. (2011). Using Arduino for Tangible Human Computer Interaction. (Tesis de Maestría), Universidad de Torino, Departamento de Informática, Italia.

Vela Pena, R. (2016). Sistema de Detección de Movimientos Basado en Sensores Inerciales Integrados. (Tesis de maestría), Instituto Politécnico Nacional, Centrode Investigación e Innovación Tecnológica, México.

Vistronica. (s.f.). Vistronica, Tienda virtual de electrónica. Recuperado el 27 de 8 de 2019, de https://www.vistronica.com/imu/ 